\title{
Characterization of Efficiently Parallel Solvable Problems on a Class of Decomposable Graphs
}

\author{
Sun-Yuan Hsieh \\ Department of Computer Science and Information Engineering \\ National Cheng Kung University \\ No 1. University Road, Tainan 701, Taiwan \\ hsiehsy@mail.ncku.edu.tw
}

\begin{abstract}
In this paper, we sketch characteristics of those problems which can be systematically solved on decomposable graphs. Trees, series-parallel graphs, outerplanar graphs, and bandwidth- $k$ graphs all belong to decomposable graphs. Let $T_{d}(|V|,|E|)$ and $P_{d}(|V|,|E|)$ denote the time complexity and processor complexity required to construct a parse tree representation $T_{G}$ for a decomposable $G=(V, E)$ on a PRAM model $M_{d}$. We define a general problem-solving paradigm to solve a wide class of subgraph optimization problems on decomposable graphs in $O\left(T_{d}(|V|,|E|)+\log \left|V\left(T_{G}\right)\right|\right)$ time using $O\left(P_{d}(|V|,|E|)+\right.$ $\left.\left|V\left(T_{G}\right)\right| / \log \left|V\left(T_{G}\right)\right|\right)$ processors on $M_{d}$. By using our paradigm, we show the following parallel complexities: (a) The maximum independent set problem on trees can be solved in $O(\log |V|)$ time using $O(|V| / \log |V|)$ processors on an EREW PRAM. (b) The maximum matching problem on series-parallel graphs can be solved in $O(\log |E|)$ time using $O(|E| / \log |E|)$ processors on an EREW PRAM. (c) The efficient domination problem on series-parallel graphs can be solved in $O(\log |E|)$ time using $O(|E| / \log |E|)$ processors on an EREW PRAM.
\end{abstract}

\section{Introduction}

A class of graphs is recursive if every graph of the class can be constructed by a finite number of applications of composition operations starting with a finite set of basis graphs. The recursive class $\Gamma$ of graphs is said to be decomposable if each graph in $\Gamma$ has a set of some specified vertices called terminals, and each composition operation is defined in terms of certain primitive operations on terminals. Trees, series-parallel graphs, outerplanar graphs, protoHalin graphs, and bandwidth- $k$ graphs are all decomposable graphs [3]. Also, every decomposable graph has a fixed upper bound on the treewidth of the graphs in the class, and graphs with treewidth at most $k$ for fixed $k$ are partial $k$-trees [8].

Properties of decomposable graphs are studied by many researchers 23178 9.1112 which resulted in sequential algorithms to solve quite a few interesting graph-theoretical problems on this special class of graphs. However, there are few results in the viewpoint of parallel computation. Given a graph problem, we say it belongs to the class of subgraph optimization problem if the object of this 
problem is to find a subgraph of the input graph to satisfy the given properties which includes an optimization constraint. For example, the problem of finding a maximum independent set is a subgraph optimization problem.

In this paper, we propose a different parallel strategy on the deterministic parallel random access machine (PRAM) [6]. Given a decomposable graph represented by its parse tree form, we define a class of subgraph optimization problems, called the $(k, \Theta)$-regular problem, and show such a class of problems can be efficiently parallelized by applying the binary tree contraction technique to the given parse tree. Let $T_{d}(|V|,|E|)$ and $P_{d}(|V|,|E|)$ denote the time complexity and processor complexity required to construct a parse tree $T_{G}$ of a decomposable graph $G=(V, E)$ on a PRAM model $M_{d}$. We show that a $(k, \Theta)$-regular problem can be solved in $O\left(T_{d}(|V|,|E|)+\log \left|V\left(T_{G}\right)\right|\right)$ time using $O\left(P_{d}(|V|,|E|)+\left|V\left(T_{G}\right)\right| / \log \left|V\left(T_{G}\right)\right|\right)$ processors on $M_{d}$. Moreover, each $(k, \Theta)$-regular problem can be solved in $O\left(\log \left|V\left(T_{G}\right)\right|\right)$ time using $O\left(\left|V\left(T_{G}\right)\right| / \log \left|V\left(T_{G}\right)\right|\right)$ processors on an EREW PRAM if $T_{G}$ is given to be an input instance. Based on the technique, we obtain the following results: (a) The maximum independent set problem on trees can be solved in $O(\log |V|)$ time using $O(|V| / \log |V|)$ processors on an EREW PRAM, (b) The maximum matching problem can be solved in $O\left(\log |E| \log ^{*}|E|\right)$ time using $O\left(|E| / \log |E| \log ^{*}|E|\right)$ processors on an EREW PRAM, and (c) The efficient domination problem on series-parallel graphs can be solved in $O\left(\log |E| \log ^{*}|E|\right)$ time using $O\left(|E| / \log |E| \log ^{*}|E|\right)$ processors on an EREW PRAM. Given a parse tree of a series-parallel graph, the problems in (b) and (c) can be optimally solved in $O(\log |E|)$ time using $O(|E| / \log |E|)$ processors on an EREW PRAM. To our knowledge, no $\mathrm{NC}$ algorithm exists for solving the problem in (c) in the literature.

\section{Preliminaries}

This paper considers finite, simple 1 and undirected graphs $G=(V, E)$, where $V$ and $E$ are the vertex and edge sets of $G$, respectively. Let $n=|V|$ and $m=|E|$. For two graphs $G_{1}=\left(V_{1}, E_{1}\right)$ and $G_{2}=\left(V_{2}, E_{2}\right)$, the union of $G_{1}$ and $G_{2}$, denoted by $G_{1} \cup G_{2}$, is the graph $\left(V_{1} \cup V_{2}, E_{1} \cup E_{2}\right)$. We say that a graph $G^{\prime}=\left(V^{\prime}, E^{\prime}\right)$ is a subgraph of $G=(V, E)$ if $V^{\prime} \subseteq V$ and $E^{\prime} \subseteq E$. Given a set $V^{\prime} \subseteq V$, the subgraph of $G$ induced by $V^{\prime}$ is the graph $G^{\prime}=\left(V^{\prime}, E^{\prime}\right)$, where $E^{\prime}=\left\{(u, v) \in E \mid u, v \in V^{\prime}\right\}$. Let $G[X]$ denote the subgraph of $G$ induced by $X \subseteq V$. For a vertex $v \in V$ of a graph $G=(V, E)$, the neighborhood of $v$ is $N_{G}(v)=\{u \in V \mid(u, v) \in E\}$ and the closed neighborhood of $v$ is $N_{G}[v]=$ $N_{G}(v) \cup\{v\}$. The subscript $G$ in the notations used in this paper can be omitted when no ambiguity arises. Given a node $v$ in a rooted tree $T$, let $T(v)$ be a subtree of $T$ rooted at $v$. For graph-theoretic terminologies and notations not mentioned here, see [5].

We follow the notations used in [8] to define the class of decomposable graphs.

\footnotetext{
${ }^{1}$ We only consider simple graphs in this paper although some of the results also apply to multigraphs.
} 
Definition 1. Let $G=(V, E, S)$ be a graph with vertex $V$, edge set $E$, and an ordered list $S$ of $t$ terminals chosen from $V$ for some fixed integer $t$. We note that the elements of $S$ are not necessary distinct.

(1) Let $B=\left\{B_{1}, B_{2}, \ldots, B_{l}\right\}$ be a finite set of basis graphs, where each $B_{i}$ is a finite graph having an ordered list of $t$ (not necessary distinct) terminals.

(2) Let $O=\left\{*_{1}, *_{2}, \ldots, *_{q}\right\}$ be a finite set of binary rules of composition, whereby two graphs $G_{i}=\left(V_{i}, E_{i}, S_{i}\right)$ and $G_{j}=\left(V_{j}, E_{j}, S_{j}\right)$ can be combined to produce new graphs $G_{i} *_{c} G_{j}, 1 \leq c \leq q$. Each rule of composition $*_{c}$ consists of three suboperations on the terminals $S_{i}$ and $S_{j}$ :

(i) Choose a subset $S_{i}{ }^{\prime}$ of distinct terminals from the list $S_{i}$ and identify each $x \in S_{i}{ }^{\prime}$ with a unique $y \in S_{j}$. Let $S_{j}{ }^{\prime}$ denote the subset of affected terminals from the list $S_{j}$.

(ii) Add any subset of the edges $\left\{(x, y) \mid x \in \overline{S_{i}{ }^{\prime}}, y \in \overline{S_{j}{ }^{\prime}}\right\}$ to $G_{i} *_{c} G_{j}$, where $\overline{S_{i}{ }^{\prime}}$ is the subset of terminals in the list $S_{i}$ but not in $S_{i}{ }^{\prime}$, and $\overline{S_{j}{ }^{\prime}}$ is defined similarly.

(iii) Select an ordered list of $t$ (not necessarily distinct) terminals from the list $S_{i}$ and $S_{j}$ to the terminals of $G_{i} *_{c} G_{j}$.

(3) The class $\Gamma$ of decomposable graphs is recursively defined as follows:

(i) Any $B_{i} \in B$ is in $\Gamma$.

(ii) If $G_{i}$ and $G_{j}$ are in $\Gamma$ and $*_{c}$ is an operation in $O$, then the graph $G_{i} *_{c} G_{j}$ is also in $\Gamma$.

Definition 2. Let $\Gamma$ be the class of decomposable graphs. The parse tree $T_{G}$ of a graph $G \in \Gamma$ is a tree in which the leaves correspond to the basis graphs from which $G$ is constructed, and each internal node represents the result of applying a composition operation to the graphs represented by the subtrees rooted at its children. Let $G_{v}$ be the subgraph of $G$ corresponding to a node $v$ of a parse tree. Note that $T_{G}(v)$ is a parse tree of $G_{v}$.

\section{A General Problem-Solving Paradigm}

\subsection{The $(k, \Theta)$-Parse Tree}

Given a graph $G$, let $\mathcal{U}_{V(G)}$ (respectively, $\mathcal{U}_{E(G)}$ ) be the set consisting of all subsets of $V(G)$ (respectively, $E(G)$ ). Given $\mathcal{Q}=\left\{Q_{1}, Q_{2}, \ldots, Q_{l}\right\}$, where $Q_{i} \in$ $\mathcal{U}_{V(G)}$ (respectively, $Q_{i} \in \mathcal{U}_{E(G)}$ ), we define $\operatorname{Min}_{v}$ (respectively, Mine) to be an operator on $\mathcal{Q}$ that returns a minimum-cardinality set $Q_{j}$ for some $1 \leq j \leq l$. The operators $\mathrm{MAX}_{v}$ (respectively, $\mathrm{MAX}_{e}$ ) can be defined similarly. For two lists $L_{1}=\left\langle l_{1}, l_{2}, \ldots, l_{i}\right\rangle$ and $L_{1}{ }^{\prime}=\left\langle l_{1}{ }^{\prime}, l_{2}{ }^{\prime}, \ldots, l_{j}{ }^{\prime}\right\rangle$, we define the concatenation of $L_{1}$ and $L_{1}{ }^{\prime}$, denoted by $L_{1} \bullet L_{1}{ }^{\prime}$, to be the list $\left\langle l_{1}, l_{2}, \ldots, l_{i}, l_{1}{ }^{\prime}, l_{2}{ }^{\prime}, \ldots, l_{j}{ }^{\prime}\right\rangle$.

Definition 3. Let $G=(V, E)$ be a decomposable graph and let $T_{G}$ be a parse tree of $G$. Given a positive integer $k$, and an operator $\Theta \in\left\{\operatorname{Min}_{v}, \operatorname{Min}_{e}, \operatorname{MAX}_{v}\right.$, $\left.\operatorname{MAx}_{e}\right\}, T_{G}$ is a $(k, \Theta)$-parse tree of $G$ if the following conditions hold. Let $v$ be a node of $T_{G}$ and let $N_{i}$ be the set of integers from 1 to $i$.

(1) If $v$ is an internal node, then it is associated with $k$ integers $a_{v, 1}, a_{v, 2}, \ldots, a_{v, k}$ 
from $N_{k}$, and the following $2 k$ functions $f_{i}:\{v\} \times N_{a_{v, i}} \mapsto N_{k}$ and $g_{i}:\{v\} \times N_{a_{v, i}} \mapsto N_{k}, 1 \leq i \leq k$.

(2) Node $v$ is also associated with a list of $k$ subgraph $2 R_{v}=$ $\left\langle R_{v, 1}, R_{v, 2}, \ldots, R_{v, k}\right\rangle$, called the target subgraphs of $v$, which are defined as follows.

CAsE 1: $v$ is a leaf. $R_{v}$ is a list of $k$ subgraphs selected from $\mathcal{U}_{V\left(G_{v}\right)}$ (respectively, $\left.\mathcal{U}_{E\left(G_{v}\right)}\right)$ if $\Theta \in\left\{\operatorname{Min}_{v}, \operatorname{MAx}_{v}\right\}$ (respectively, $\Theta \in\left\{\operatorname{Min}_{e}, \operatorname{Max}_{e}\right\}$ ).

CASE 2: $v$ is an internal node. Let $u$ and $w$ be two children of $v$. Then,

$R_{v, i}=\Theta\left\{R_{u, f_{i}(u, 1)} \cup R_{w, g_{i}(w, 1)}, R_{u, f_{i}(u, 2)} \cup R_{w, g_{i}(w, 2)}, \ldots, R_{u, f_{i}\left(u, a_{v, i}\right)} \cup\right.$ $\left.R_{w, g_{i}\left(w, a_{v}, i\right.}\right\}$, where $1 \leq i \leq k$.

Definition 4. Let $T_{G}$ be a $(k, \Theta)$-parse tree. The $(k, \Theta)$-parse tree problem is the problem to find the $k$ target subgraphs of the root of $T_{G}$.

Lemma 1. The $(k, \Theta)$-parse tree problem can be solved in $O\left(k^{2} n\right)$ time, where $n$ is the number of vertices of the given tree.

\subsection{Parallel Complexities of the $(k, \Theta)$-Regular Problem}

In this section, we apply the binary tree contraction technique described in [1] to parallelize the $(k, \Theta)$-regular problem. This technique recursively applies two operations, prune and bypass, to a given binary tree. Prune $(u)$ is an operation which removes a leaf node $u$ from the current tree, and bypass $(v)$ is an operation (following a prune operation) that removes a node $v$ with exactly one child $w$ and then lets the parent of $v$ become the new parent of $w$. We define a contraction phase to be the consecutively execution of prune and bypass operations.

Let $T$ be an $n$-leave binary tree with the root $r$. Given a Euler tour starting from $r$ of $T$, the algorithm initially numbers the leaves from 1 to $n$ according to the order of their appearances in the tour. Then, the algorithm repeats the following steps. In each step, prune and bypass work only on the leaves with odd index and their parents. Hence, these two operations can be performed independently and delete $\left\lfloor\frac{l}{2}\right\rfloor$ leaves together with their parents on the binary tree in each step, where $l$ is the number of the current leaves. Therefore, the tree will be reduced to a three-node tree after repeating the steps in $\lceil\log n\rceil$ times.

Lemma 2. [1] If the prune operation and bypass operation can be performed by one processor in constant time, the binary tree contraction algorithm can be implemented in $O(\log n)$ time using $O(n / \log n)$ processors on an EREW PRAM, where $n$ is the number of nodes in an input binary tree.

Consider a node $x$ in a rooted tree $T$. Any node $y$ on the unique path from $x$ to the root is called an ancestor of $x$. If $y$ is an ancestor of $x$, then $x$ is a descendant of $y$. Further, $x$ is a proper descendant of $y$ when $x \neq y$. Note that every node is both an ancestor and a descendant of itself. For convenience, we allow $\mathcal{U}_{G}$ to represent one of $\mathcal{U}_{V(G)}$ and $\mathcal{U}_{E(G)}$ if it is not particularly specified.

\footnotetext{
${ }^{2}$ In this paper, a subgraph $H$ of $G$ is represented by a set $Q$ : If $Q \in \mathcal{U}_{V(G)}$, then $H=(Q, \emptyset)$; If $Q \in \mathcal{U}_{E(G)}$, then $H=(\{x \mid x$ is an endpoint of an edge in $Q\}, Q)$.
} 
Definition 5. Let $u$ and $v$ be two nodes of a $(k, \Theta)$-parse tree $T$ such that $u$ is a descendant of $v$. A $k$-ary function $h: \mathcal{U}_{G_{u}}{ }^{k} \mapsto \mathcal{U}_{G_{v}}$ possesses the canonical form, if $h\left(X_{1}, \ldots, X_{k}\right)=\Theta\left\{X_{b_{1}} \cup C_{1}, X_{b_{2}} \cup C_{2}, \ldots, X_{b_{a}} \cup C_{a}\right\}$, where $b_{i} \neq b_{j}$ for two distinct $1 \leq i, j \leq a$, and $C_{i} \in\left(\mathcal{U}_{G_{v}} \backslash \mathcal{U}_{G_{u}}\right)$.

The following lemma can be shown by the set theory and properties of the function composition.

Lemma 3. Let $\Theta \in\left\{\mathrm{MIN}_{v}, \operatorname{Min}_{e} \operatorname{MAx}_{v}, \operatorname{MAX}_{e}\right\}$, and let $h_{0}: \mathcal{U}_{G_{u}}{ }^{k} \mapsto \mathcal{U}_{G_{v}}$ be a function with the canonical form, where $u$ is a descendant of $v$. If $k$ functions $h_{i}: \mathcal{U}_{G_{w}}{ }^{k} \mapsto \mathcal{U}_{G_{u}}$ possess the canonical form, where $1 \leq i \leq k$ and $w$ is a descendant of $u$, then the function obtained from the composition $h_{0} \circ\left(h_{1}, h_{2}, \ldots, h_{k}\right): \mathcal{U}_{G_{w}}{ }^{k} \mapsto \mathcal{U}_{G_{v}}$ possesses the canonical form.

We next develop a parallel algorithm for the $(k, \Theta)$-parse tree problem. For a node $x$ in the current tree $H$, let $\operatorname{par}_{H}(x)$ (respectively, $\operatorname{chil}_{H}(x)$ ) denote the parent (children) of $x$, and let $\operatorname{sib}_{H}(x)$ denote the sibling of $x$. The subscript $H$ can be omitted if no ambiguity arises. Recall that $H(x)$ be the subtree of $H$ rooted at $x$, and $R_{x}=\left\langle R_{x, 1}, \ldots, R_{x, k}\right\rangle$ is the list of the target subgraphs associated with $x$.

During the process of executing the tree contraction, we aim at constructing $k$ $k$-ary functions $h_{x, 1}, h_{x, 2}, \ldots, h_{x, k}$ associated with each node $x$ of the current tree such that $h_{x, i}$ 's possess the canonical form and satisfy the condition described below. Let $v$ be an internal node in the current tree whose left child and right child are $u$ and $w$, respectively. Also let $u^{\prime}$ be the left child and $w^{\prime}$ be the right child of $v$ in the original tree. For the remainder of this section, we call $u^{\prime}$ and $w^{\prime}$ replacing ancestors of $u$ and $w$ with respect to $v$, respectively. Once $R_{u, i}$ and $R_{w, i}, 1 \leq i \leq k$, are provided as the inputs of $h_{u, i}$ and $h_{w, i}$, respectively, the target subgraphs of $v$ can be obtained from $R_{u^{\prime}}=\left\langle R_{u^{\prime}, 1}, \ldots, R_{u^{\prime}, k}\right\rangle=$ $\left\langle h_{u, 1}\left(R_{u, 1}, \ldots, R_{u, k}\right), \ldots, h_{u, k}\left(R_{u, 1}, \ldots, R_{u, k}\right)\right\rangle$, and $R_{w^{\prime}}=\left\langle R_{w^{\prime}, 1}, \ldots, R_{w^{\prime}, k}\right\rangle=$ $\left\langle h_{w, 1}\left(R_{w, 1}, \ldots, R_{w, k}\right), \ldots, h_{w, k}\left(R_{w, 1}, \ldots, R_{w, k}\right)\right\rangle$, using the formula

$$
\begin{aligned}
R_{v, i}= & \Theta\left\{R_{u^{\prime}, f_{i}\left(u^{\prime}, 1\right)} \cup R_{w^{\prime}, g_{i}\left(w^{\prime}, 1\right)}, R_{u^{\prime}, f_{i}\left(u^{\prime}, 2\right)} \cup R_{w^{\prime}, g_{i}\left(w^{\prime}, 2\right)}, \ldots, R_{u^{\prime}, f_{i}\left(u^{\prime}, a_{v, i}\right)} \cup\right. \\
& \left.R_{w^{\prime}, g_{i}\left(w^{\prime}, a_{v, i}\right)}\right\} .
\end{aligned}
$$

where, $\quad R_{u^{\prime}, f_{i}\left(u^{\prime}, j\right)}=h_{u, f_{i}\left(u^{\prime}, j\right)}\left(R_{u, 1}, \ldots, R_{u, k}\right) \quad$ and $\quad R_{w^{\prime}, g_{i}\left(w^{\prime}, j\right)}=$ $h_{w, g_{i}\left(w^{\prime}, j\right)}\left(R_{w, 1}\right.$, $\left.\ldots, R_{w, k}\right)$ for $1 \leq j \leq a_{v, i}$.

We call the functions $h_{x, i}, 1 \leq i \leq k$, computed for each node $x$ in the current tree the crucial functions of $x$.

We next describe the details of our algorithm. Initially, for each node $v$ in the given tree we construct $k$ functions $h_{v, i}\left(X_{1}, \ldots, X_{k}\right)=\Theta\left\{X_{i} \cup \emptyset\right\}, 1 \leq i \leq k$. Clearly, these functions are crucial functions.

In the execution of the tree contraction, assume that prune $(u)$ and $\operatorname{bypass}(\operatorname{par}(u))$ are performed consecutively. Let $\operatorname{par}(u)=v$ and $\operatorname{sib}(u)=w$ in the current tree. Let $u^{\prime}$ and $w^{\prime}$ be the replacing ancestors of $u$ and $w$ with respect to $v$, respectively. Assume that $h_{u, i}$ and $h_{w, i}, 1 \leq i \leq k$, are crucial functions of $u$ 
and $w$ in the current tree. Thus $R_{u^{\prime}}=\left\langle h_{u, 1}\left(R_{u, 1}, \ldots, R_{u, k}\right), \ldots, h_{u, k}\left(R_{u, 1}, \ldots\right.\right.$, $\left.\left.R_{u, k}\right)\right\rangle$ and $R_{w^{\prime}}=\left\langle h_{w, 1}\left(R_{w, 1}, \ldots, R_{w, k}\right), \ldots, h_{w, k}\left(R_{w, 1}, \ldots, R_{w, k}\right)\right\rangle$. Since $u$ is a leaf, $R_{u, i}$ 's are associated with $u$ before executing the tree contraction algorithm. Therefore, the above $k$ target subgraphs $R_{u^{\prime}}$ can be obtained through function evaluation. On the other hand, since $w$ is not a leaf in the current tree, $R_{w, i}$, $1 \leq i \leq k$, is an indeterminate value represented by variable $X_{i}$. Hence. $R_{w^{\prime}}$ can be represented by $\left\langle h_{w, 1}\left(X_{1}, \ldots, X_{k}\right), \ldots, h_{w, k}\left(X_{1}, \ldots, X_{k}\right)\right\rangle$. By Equation 1 we construct $k$ intermediate functions representing $k$ target subgraphs $R_{v}$ from $R_{u^{\prime}}$ and $R_{w^{\prime}}$ by:

$$
\begin{aligned}
R_{v, i}= & \Theta\left\{R_{u^{\prime}, f_{i}\left(u^{\prime}, 1\right)} \cup R_{w^{\prime}, g_{i}\left(w^{\prime}, 1\right)}, R_{u^{\prime}, f_{i}\left(u^{\prime}, 2\right)} \cup R_{w^{\prime}, g_{i}\left(w^{\prime}, 2\right)}, \ldots, R_{u^{\prime}, f_{i}\left(u^{\prime}, a_{v, i}\right)} \cup\right. \\
& \left.R_{w^{\prime}, g_{i}\left(w^{\prime}, a_{v, i}\right)}\right\}
\end{aligned}
$$

where $R_{w^{\prime}, g_{i}\left(w^{\prime}, j\right)}=h_{w, g_{i}\left(w^{\prime}, j\right)}\left(X_{1}, \ldots, X_{k}\right), 1 \leq j \leq a_{v, i}$

As with the proof similar to that of Lemma 3, Equation 2 can be further simplified as

$$
R_{v, i}=\Theta\left\{X_{b_{1}} \cup C_{1}, X_{b_{2}} \cup C_{2}, \ldots, X_{b_{a}} \cup C_{a}\right\},
$$

where $b_{i} \neq b_{j}$ for two distinct $1 \leq i, j \leq a, X_{b_{i}}$ are variables drawn from $\mathcal{U}_{w}$, and $C_{i} \in\left(\mathcal{U}_{G_{v}} \backslash \mathcal{U}_{G_{w}}\right)$.

Therefore, the above functions (constructed after executing prune $(u)$ ) possess the canonical form. Given those functions $R_{v, i}$ 's, the contribution to the $k$ target subgraphs of $\operatorname{par}(v)$ is obtained by function composition $h_{v, i}\left(R_{v, 1}, \ldots, R_{v, k}\right)$ for all $1 \leq i \leq k$. These functions are constructed for $w$ after executing bypass $(\operatorname{par}(v))$. By Lemma 3, $h_{v, i}\left(R_{v, 1}, \ldots, R_{v, k}\right), 1 \leq i \leq k$, possesses the canonical form. Hence, we have the following lemma.

Lemma 4. During the process of executing the binary tree contraction on a $(k, \Theta)$-parse tree to remove some nodes, the crucial functions of the remaining nodes of the current tree can be constructed in $O\left(k^{3}\right)$ time using one processor.

Theorem 1. The $(k, \Theta)$-parse tree problem can be solved in $O\left(k^{3} \log n\right)$ time using $O(n / \log n)$ processors on an EREW PRAM, where $n$ is the number of nodes of the input tree.

Definition 6. Let $G$ be a decomposable graph and let $T_{G}$ be a parse tree. A problem $\mathcal{P}$ is said to be a $(k, \Theta)$-regular problem on $G$ if $\mathcal{P}$ can be reduced to a $(k, \Theta)$-parse tree problem $\mathcal{B}$ on $T_{G}$ such that the solution of $\mathcal{B}$ is exactly the solution of $\mathcal{P}$. Moreover, the reduction scheme takes $O\left(k^{3} \log \left|V\left(T_{G}\right)\right|\right)$ time using $O\left(\left|V\left(T_{G}\right)\right| / \log \left|V\left(T_{G}\right)\right|\right)$ processors on an EREW PRAM.

Note that each $(k, \Theta)$-regular problem corresponds to a $(k, \Theta)$-parse tree. This tree is obtained from a parse tree $T_{G}$ in which some additional data structures are associated with $V\left(T_{G}\right)$ (refer to Definition 3). In Section 4 we assume that a parse tree is given for solving a $(k, \Theta)$-regular problem on a decomposable graph.

The following result directly follows from Definition 6] and Theorem 1 . 
Theorem 2. Given a parse tree of a decomposable graph $G, a(k, \Theta)$ regular problem on $G$ can be solved in $O\left(k^{3} \log \left|V\left(T_{G}\right)\right|\right)$ time using $O\left(\left|V\left(T_{G}\right)\right| / \log \left|V\left(T_{G}\right)\right|\right)$ processors on an EREW PRAM.

Corollary 1. $A(k, \Theta)$-regular problem of a decomposable graph $G=(V, E)$ can be solved in $O\left(T_{d}(|V|,|E|)+\log \left|V\left(T_{G}\right)\right|\right)$ time using $O\left(P_{d}(|V|,|E|)+\right.$ $\left.\left|V\left(T_{G}\right)\right| / \log \left|V\left(T_{G}\right)\right|\right)$ processors on $M_{d}$.

\section{$4(k, \Theta)$-Regular Problems}

Given a problem $\mathcal{P}$, a graph $G_{1}$, a subgraph $G_{2}$ of $G_{1}$, and a subset $Q$ of vertices in $G_{2}, \mathcal{P}_{Q}\left(G_{1}, G_{2}\right)$ is a solution to the input graph $G_{1}$ such that this solution contains all vertices in $Q$ and is in $G_{2}$. For the case of $Q=\emptyset$, i.e., $\mathcal{P}_{\emptyset}\left(G_{1}, G_{2}\right)$, the notation represents a solution to $G_{1}$ and this solution is contained in $G_{2}$. For brevity, let $\mathcal{P}_{Q}(G, G)=\mathcal{P}_{Q}(G)$.

An independent set of a graph is a subset of its vertices such that no two vertices in the subset are adjacent. The maximum independent set problem $\mathcal{I}$ is the problem of finding a maximum-cardinality independent set in the input graph. Using our notation, given an input graph $G$, a solution is $\mathcal{I}_{\emptyset}(G)$. For a basis rooted tree $G=(\{r\},\{\},(r)), \mathcal{I}_{\emptyset}(G)$ and $\mathcal{I}_{\{r\}}(G)$ are both equal to $\{r\}$, and $\mathcal{I}_{\emptyset}(G[V \backslash\{r\}])=\emptyset$.

\section{Lemma 5.}

Assume $G=\left(V_{1} \cup V_{2}, E_{1} \cup E_{2} \cup\left\{\left(r_{1}, r_{2}\right)\right\},\left(r_{1}\right)\right)$ is obtained from $G_{1}=$ $\left(V_{1}, E_{1},\left(r_{1}\right)\right)$ and $G_{2}=\left(V_{2}, E_{2},\left(r_{2}\right)\right)$.

(1) $\mathcal{I}_{\emptyset}(G)=\operatorname{MAx}_{v}\left\{\mathcal{I}_{\left\{r_{1}\right\}}\left(G_{1}\right) \cup \mathcal{I}_{\emptyset}\left(G_{2}\left[V_{2} \backslash\left\{r_{2}\right\}\right]\right)\right.$,

$\left.\mathcal{I}_{\emptyset}\left(G_{1}\left[V_{1} \backslash\left\{r_{1}\right\}\right]\right) \cup \mathcal{I}_{\left\{r_{2}\right\}}\left(G_{2}\right), \mathcal{I}_{\emptyset}\left(G_{1}\left[V_{1} \backslash\left\{r_{1}\right\}\right]\right) \cup \mathcal{I}_{\emptyset}\left(G_{2}\left[V_{2} \backslash\left\{r_{2}\right\}\right]\right)\right\} ;$

(2) $\mathcal{I}_{\{r\}}(G)=\mathcal{I}_{\left\{r_{1}\right\}}\left(G_{1}\right) \cup \mathcal{I}_{\emptyset}\left(G_{2}\left[V_{2} \backslash\left\{r_{2}\right\}\right]\right)$;

(3) $\mathcal{I}_{\emptyset}(G[V \backslash\{r\}])=\mathcal{I}_{\emptyset}\left(G_{1}\left[V_{1} \backslash\left\{r_{1}\right\}\right]\right) \cup \mathcal{I}_{\emptyset}\left(G_{2}\right)$.

Proof. Straightforward.

By the above result, it is not difficult to obtain the following two theorems.

Theorem 3. The maximum independent set problem is a $\left(3, \mathrm{MAX}_{v}\right)$-regular problem on trees.

Theorem 4. The maximum independent set problem on trees can be solved in $O(\log n)$ time using $O(n / \log n)$ processors on an EREW PRAM, where $n$ is the number of vertices of the input graph.

Given an undirected graph $G=(V, E)$, a matching is a subset of edges $M \subseteq E$ such that for all vertices $v \in V$, at most one edge of $M$ is incident on $v$. The maximum matching problem $\mathcal{M}$ is the problem of finding a matching of maximum cardinality. For a basis series-parallel graph $G=(\{l, r\},\{(l, r)\},(l, r))$, $\mathcal{M}_{\emptyset}(G)=\{(l, r)\}, \mathcal{M}_{\{l\}}(G[V \backslash\{r\}])=\emptyset, \mathcal{M}_{\{r\}}(G[V \backslash\{l\}])=\emptyset, \mathcal{M}_{\{l, r\}}(G)=$ $\{(l, r)\}, \mathcal{M}_{\emptyset}(G[V \backslash\{l, r\}])=\emptyset$. We can further show that the maximum matching problem is a $\left(5\right.$, MAx $\left._{e}\right)$-regular problem on series-parallel graphs.

By the methods described in [4,10] to construct parse trees of series-parallel graphs, we have the following theorem. 
Theorem 5. The maximum matching problem on series-parallel graphs can be solved in sequential $O(n+m)$ time, and in parallel in $O\left(\log m \log ^{*} m\right)$ time using $O\left(m / \log m \log ^{*} m\right)$ processors on an EREW PRAM.

Given a simple graph $G=(V, E)$, a vertex $v \in V$ is said to dominate itself and all vertices adjacent to $v$. A subset $D$ of $V$ is called an efficient dominating set of $G$ if every vertex in $V$ is dominated by exactly one vertex in $D$. Note that not all graphs have efficient dominating sets. Moreover, if a graph possesses an efficient dominating set, then all these sets have the same cardinality. The efficient domination problem $\mathcal{D}$ is the problem to find an efficient dominating set of a given graph if such a set exists. Using our paradigm, we can also show the following result.

Theorem 6. The efficient domination problem on series-parallel graphs can be solved in linear $O(n+m)$ time, and in parallel in $O\left(\log m \log ^{*} m\right)$ time using $O\left(m / \log m \log ^{*} m\right)$ processors on an EREW PRAM.

\section{References}

1. K. Abrahamson, N. Dadoun, D. G. Kirkpatrick, and T. Przytycka, A simple parallel tree contraction algorithm, Journal of Algorithms, 10, pp. 287-302, 1989.

2. S. Arnborg and A. Proskurowski, Linear time algorithms for NP-hard problems restricted to partial $k$-trees, Discrete Applied Mathematics, 23, pp. 11-24, 1989.

3. M. W. Bern, E. L. Lawler, and A. L. Wong, Linear-time computation of optimal subgraphs of decomposable graphs, Journal of Algorithms, 8:216-235, 1987.

4. H. L. Bodlaender and B. van Antwerpen-de Fluiter, Parallel algorithms for series parallel graphs and graphs with treewidth two, Algorithmica, 29(4):534-559, 2001.

5. M. C. Golumbic, Algorithmic graph theory and perfect graphs, Academic press, New York, 1980.

6. R. M. Karp and V. Ramachandran, Parallel algorithms for shared memory machines, Handbook of Theoretical Computer Science, North-Holland, Amsterdan, pp. 869-941, 1990.

7. S. Mahajan and J. G. Peters, Algorithms for regular properties in recursive graphs, in: Proceedings of the 25th Allerton Conference on Communication, Control, and Computing, pp. 14-23, 1987.

8. S. Mahajan and J. G. Peters, Regularity and locality in $k$-terminal graphs, Discrete Applied Mathematics, 54:229-250, 1994.

9. K. Takamizawa, T. Nishizeki, and N. Saito, Linear-time computability of combinatorial problems on series-parallel graphs, Journal of the ACM, 29:623-641, 1982.

10. J. Valdes, R. E. Tarjan, and E. L. Lawler, The recognition of series-parallel digraphs, SIAM Journal on Computing, 11:298-313, 1982.

11. T. V. Wimer, Linear algorithms on $k$-terminal graphs, Ph.D. Thesis, Clemson University, Clemson, SC, 1987.

12. T. V. Wimer and S. T. Hedetniemi, $K$-terminal recursive families of graphs, Congressus Numerantium, 63:161-176, 1988. 